Vietnam Journal of Mechanics, VAST, Vol.43, No. 1 (2021), pp. $27-41$

DOI: https://doi.org/10.15625/0866-7136/15332

\title{
AN IMPROVED MESHLESS METHOD FOR FINITE DEFORMATION PROBLEM IN COMPRESSIBLE HYPERELASTIC MEDIA
}

\author{
Nha Thanh Nguyen ${ }^{1,2, *}$, Minh Ngoc Nguyen ${ }^{1,2}$, Thien Tich Truong ${ }^{1,2}$, Tinh Quoc Bui ${ }^{3}$ \\ ${ }^{1}$ Department of Engineering Mechanics, Faculty of Applied Science, \\ Ho Chi Minh City University of Technology (HCMUT), Vietnam \\ ${ }^{2}$ Vietnam National University Ho Chi Minh City, Vietnam \\ ${ }^{3}$ Department of Civil and Environmental Engineering, Tokyo Institute of Technology, Japan \\ *E-mail: nhanguyen@hcmut.edu.vn
}

Received: 03 August 2020 / Published online: 01 February 2021

\begin{abstract}
Hyperelastic materials are considered as special category of elastic solid materials because of their nonlinear complicated constitutive laws. Due to large strain state, the behaviour of such materials is often considered in finite deformation analysis. The nonlinear large deformation behavior of such materials is important. In this study, a novel meshless radial point interpolation method (RPIM) enhanced by Cartesian transformation method (CTM), an effective numerical integration, is presented for nonlinear behavior of hyperelastic media under finite deformation state with total Lagrange formulation. Unlike the mesh-based approaches, the meshless methods have shown their advantages in analysis of large deformation problems. The developed CTM-based RPIM is thus free from the need for background cells, which are often used for numerical integration in many conventional meshfree approaches. The developed meshfree method owns some desirable features of an effective technique in solving large deformation, which will be illustrated through the numerical experiments in which our computed results are validated against reference solutions derived from other approaches.
\end{abstract}

Keywords: CTM-based meshless RPIM, hyperelastic materials, large deformation.

\section{INTRODUCTION}

Hyperelastic materials have attracted researchers from both industrial and scientific communities due to their widely application fields. Rubber-like materials have good shock-absorbing characteristics, lightweight and good form-ability so they have been widely applied to shin-guards, shoe-soles and protectors as shock-absorbing materials in sport tools and accessories. To ensure the strength and duration of part or structure made of these mentioned materials, it is necessary to consider finite deformation analyses. For most of cases, it is impossible to find an analytical method to solve these highly 
non-linear problems. In the other hand, experiments can help testing the behaviour of hyperelastic product but they are expensive and time consuming. Along with the developing of computational engineering, the finite element methods (FEM) are widely used for simulating the non-linear elastic problems [1-4]. However, FEM and mesh-based methods are limitative in certain cases. One example is when large deformation cannot be ignored. The standard element has to be convexed in shape in order to provide good approximated solution. When large deformations occur, some elements could be distorted such that convexity is lost and numerical errors add up considerably. Moreover, the large strain can cause high aspect ratio or high skewness in both quadrilateral and triangular element types.

To overcome the disadvantages of mesh-based methods, the meshfree methods have been proposed to alleviate the difficulties related to finite element mesh [5-9]. Meshfree methods do not require finite element for discretizing the problem domain. Instead, the problem domain is represented only by scattered nodes, including nodes on boundaries and nodes inside the domain. When there is a change in domain geometry such as crack propagation or large deformation problems, nodes could be added, removed or updated position without difficulties. To apply for large deformation problems, there are some meshless approaches have been presented. Gu et al. [10] introduced a meshless local Kriging method for large deformation analyses. In the same year, a meshless local Petrov-Galerkin method was presented for large deformation contact analysis of elastomers by the group of $\mathrm{Hu}$ [11]. To simulate the dynamic behavior of hyperelastic model, [12] used local Petrov-Galerkin method for impact problems of such material. Zang et al. [13] proposed a combination of FEM and element-free Galerkin method for topology optimization problem of hyperelastic material. Recently, a strongform meshfree method was presented by [14] for stress analysis of hyperelastic models. However, traditional meshfree methods still have lititations, one of them is the requirement of "background cells" for numerical integration. To overcome this restriction, Khosravifard and Hematiyan presented the "Cartesian Transformation Method" (CTM) [15] to perform integrals without using integration cells and apply for non-linear transient heat conduction analysis of functionally graded materials [16]. CTM technique made the meshfree methods become free from background mesh. This method was also presented for dynamic analysis of sandwich beams with functionally graded core [17]. Recently, the extended meshless radial point interpolation method (XRPIM) was improved by the present authors in which XRPIM associated with CTM technique was first presented for linear thermal crack propagation problem with isotropic material [18]. In this study, the CTM-based RPIM is continue developed and presented for the first time for 2D non-linear large deformation behavior of hyperelastic material. The obtained results are verified with reference solution.

The paper is organized as follows. Right after this Introduction part is a brief review on hyperelastic material model in Section 2. Next, the explanation for improved RPIM method using CTM technique is shown in Section 3. The numerical examples and obtained results are discussed in Section 4. Finally, Section 5 presents main conclusions and remarks about the proposed method. 


\section{CONSTITUTIVE LAW FOR HYPERELASTIC PROBLEMS}

Considering a 2D hyperelastic model that undergoes the finite deformation, the strain energy function $W$ can be stated as a function of the right Cauchy-Green strain tensor $\mathbf{C}$ and the stress is derived from that strain energy function. The deformation gradient tensor $\mathbf{F}$ in the current configuration is defined as

$$
\mathbf{F}=\frac{\partial u_{i}}{\partial X_{j}}+\delta_{i j}
$$

The right Cauchy-Green tensor, the second Piola-Kirchoff tensor $\mathbf{S}$ and the real stress (Cauchy stress) $[19,20]$ can be written as

$$
\mathbf{C}=\mathbf{F}^{T} \mathbf{F} ; \quad \mathbf{S}=2 \frac{\partial W}{\partial \mathbf{C}} ; \quad \sigma=\frac{1}{J} \mathbf{F S F}^{T},
$$

where $J$ is the determinant of the deformation gradient tensor $\mathbf{F}$.

In this study, the compressible neo-Hookean model is adopted [20], the strain energy density function of such material is suitable with experiment with medium elastic strains, the relationship is given by

$$
W(\mathbf{C})=\frac{K}{2}(\ln J)^{2}+\frac{\mu}{2}\left(I_{1}-2 \ln J-3\right),
$$

where $\mu$ and $K$ are the shear modulus and bulk modulus, respectively. $I_{1}$ is the first invariant of the right Cauchy-Green tensor $\mathbf{C}$ and $J$ parameter relates with the third invariant of $\mathbf{C}$ tensor

$$
I_{1}=\operatorname{tr} \mathbf{C} ; I_{3}=J^{2}=(\operatorname{det} \mathbf{F})^{2}=\operatorname{det} \mathbf{C} .
$$

From Eqs. (2) and (3), the constitutive law for nonlinear stress-strain relation in compressible neo-Hookean material is obtained as

$$
\mathbf{S}=K \ln J(\mathbf{C})^{-1}+\mu\left(\mathbf{I}-\mathbf{C}^{-1}\right)
$$

\section{CTM-BASED RPIM METHOD FOR HYPERELASTIC BEHAVIOR}

\subsection{Weak form and discretized formulations}

Consider a continuum domain $\Omega$ made from hyperelastic material as shown in Fig. 1, the body is subjected to a traction $\overline{\mathbf{t}}$ on boundary $\Gamma_{t}$, body force $\overline{\mathbf{b}}$ and displacement $\overline{\mathbf{u}}$ on boundary $\Gamma_{u}$. The general weak form for nonlinear problem defined for the deformed domain $\phi(\Omega)$ in the current configuration is given by

$$
\int_{\phi(\Omega)} \sigma: \nabla \delta \mathbf{u} d V-\int_{\phi(\Omega)} \delta \mathbf{u} \cdot \overline{\mathbf{b}} d V-\int_{\phi\left(\Gamma_{t}\right)} \delta \mathbf{u} \cdot \overline{\mathbf{t}} d \Gamma=0,
$$

where $\sigma$ is the Cauchy stress and $\delta \mathbf{u}$ is the vector of test functions.

In this study, the meshless RPIM that is developed basing on the Galerkin weak form and discretization procedure [21,22]. The displacement field $\mathbf{u}$ is approximated by the use of RPIM shape functions $\boldsymbol{\Phi}$ and nodal displacement vector $\mathbf{u}_{s p}$ in a support domain. The 


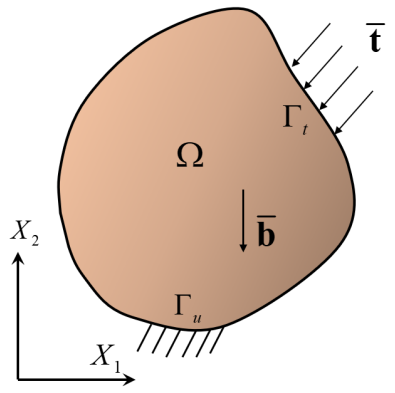

Fig. 1. Hyperelastic model with boundary conditions

approximated displacement, its variational and the gradient of test functions are written as

$$
\mathbf{u}=\boldsymbol{\Phi} \mathbf{u}_{s p} ; \quad \delta \mathbf{u}=\boldsymbol{\Phi} \delta \mathbf{u}_{s p} ; \quad \nabla \delta \mathbf{u}=\mathbf{B} \delta \mathbf{u}_{s p},
$$

where $\mathbf{B}$ is the B-operator, this matrix contents the derivatives of shape functions with respect to the current configuration.

Substituting Eq. (7) into Eq. (6), the weak form can be rewritten as follow

$$
\begin{gathered}
\int_{\phi(\Omega)} \mathbf{B}^{T} \sigma d V-\int_{\phi(\Omega)} \boldsymbol{\Phi}^{T} \overline{\mathbf{b}} d V-\int_{\phi\left(\Gamma_{t}\right)} \boldsymbol{\Phi}^{T} \overline{\mathbf{t}} d \Gamma=0, \\
\text { or } \quad \mathbf{R}(\mathbf{u})=\mathbf{F}_{\text {int }}-\mathbf{F}_{\text {ext }}=0,
\end{gathered}
$$

where $\mathbf{R}(\mathbf{u})$ denotes the residual vector that is the function of displacement field.

Apply the linearization procedure to Eq. (8) or Eq. (9), obtain the two nonlinear parts for large deformation hyperelastic problem $[23,24]$

$$
\Delta \mathbf{R}(\mathbf{u})=\int_{\phi(\Omega)} \Delta \mathbf{B}^{T} \sigma d V+\int_{\phi(\Omega)} \mathbf{B}^{T} \Delta \sigma d V=\mathbf{K}_{t a n} \Delta \mathbf{u} .
$$

The first part in Eq. (10) relates to the stress state of the current configuration, so it represents for the geometric nonlinear of the problem. The second one shows the variation of the stress and it stands for the material nonlinear of the model. Finally, the tangent stiffness matrix in Eq. (10) can be written as

$$
\mathbf{K}_{t a n}=\int_{\phi(\Omega)} \mathbf{G}^{T} \mathbf{M G} d V+\int_{\phi(\Omega)} \mathbf{B}^{T} \Delta \mathbf{C}^{\mathbf{e}} \mathbf{B} d V,
$$

where the matrix $\mathbf{M}$ and matrix $\mathbf{G}$ at node $i$ are defined as

$$
\mathbf{M}=\sigma \otimes \mathbf{I}_{2 \times 2} ; \quad \mathbf{G}^{I}=\left[\begin{array}{l}
\frac{\partial \phi_{i}}{\partial x} \mathbf{I}_{2 \times 2} \\
\frac{\partial \phi_{i}}{\partial y} \mathbf{I}_{2 \times 2}
\end{array}\right] .
$$

where $\mathbf{I}_{2 \times 2}$ is the $(2 \times 2)$ identity matrix. 


\subsection{CTM-based meshless RPIM method}

In this study, the RPIM is used for the definition of meshfree shape functions. The procedure for the construction of RPIM shap functions is described in [21,22]. Similar to the conventional element free Galerkin (EFG) method, the meshless RPIM approach is also based on the global weak form. The most advantage of RPIM shape function is its satisfaction of Kronecker delta property, this leads to convenience in treatment of boundary conditions. However, if the traditional RPIM method is used, the integrals in Eq. (6) or Eq. (11) are still conducted with the help of background cells. In this proposed approach, the CTM is chosen to alternate the Gaussian integration. Suppose that a 2D domain integral is to be calculated

$$
I=\int_{\Omega} f(x, y) d \Omega,
$$

where $f(x, y)$ is an arbitrary function. Let $\Omega_{A}$ be the auxiliary domain that contains the domain $\Omega$ and the new function $g(x, y)$ is defined over the domain $\Omega_{A}$ as

$$
g(x, y)= \begin{cases}f(x, y), & (x, y) \in \Omega \\ 0, & (x, y) \notin \Omega\end{cases}
$$

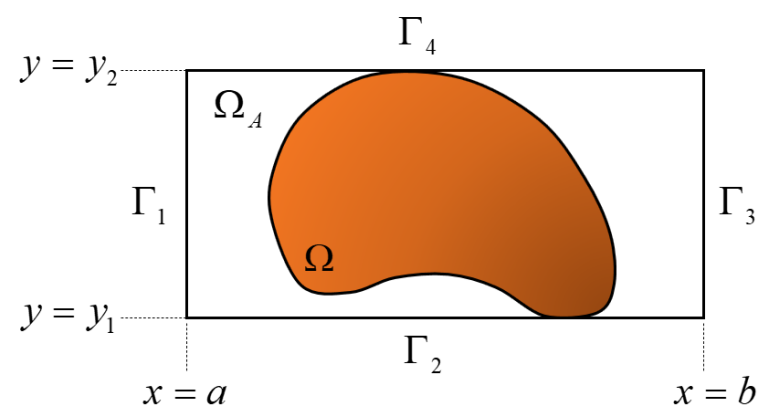

Fig. 2. An auxiliary domain $\Omega_{A}$ containing domain $\Omega$

In this study, a rectangle is used as an auxiliary domain (see Fig. 2), the domain integral in Eq. (13) can be rewritten as a double boundary integral

$$
I=\int_{y_{1}}^{y_{2}}\left[\int_{a}^{b} g(x, y) d x\right] d y=\int_{y_{1}}^{y_{2}} h(y) d y,
$$

where $h(y)=\int_{a}^{b} g(x, y) d x$.

To calculate the integral in Eq. (15), the boundaries $\Gamma_{1}$ and $\Gamma_{3}$ are divided into $n$ integration intervals and the Gaussian quadrature method is used. The integral $I$ in Eq. (15) can be computed as

$$
I=\sum_{i=1}^{n}\left(\int_{y_{i}}^{y_{i+1}} h(y) d y\right)=\sum_{i=1}^{n}\left(\int_{-1}^{1} h(\eta) J d \eta\right)=\sum_{i=1}^{n} \sum_{j=1}^{m} h\left(\eta_{j}\right) J w_{j},
$$


where $\eta_{j}$ is Gaussian points, $w_{j}$ is weight numbers and $J=d y / d \eta=\left(y_{i+1}-y_{i}\right) / 2$ denotes the Jacobian of the 1D transformation.

In numerical practice, to perform the integrals, a system of CTM integration points is generated on the rectangular auxiliary domain $\Omega_{A}$ and a simple algorithm is applied to detect if an integration point belongs to the domain or not. In CTM-based meshless method, there is no 2D integration cell created. This is the advantage of CTM approach because meshing is a time-consuming task and CTM can make the RPIM method become a truly meshless method. The detail procedure for CTM technique can be found in papers [15-18].

\section{NUMERICAL EXAMPLES}

\subsection{Cook's membrane problem}

The first numerical example for finite deformation analysis deals with a popular benchmark problem, Cook's membrane, which aims to show the accuracy of the developed CTM-based RPIM. As shown in Fig. 3(a), the model has a trapezoidal shape that is clamped at the left edge and loaded by a traction force along the right edge. The compressible neo-Hookean model is used for the hyperelastic material of the problem, the shear modulus is $\mu=80.194 \mathrm{~N} / \mathrm{mm}^{2}$ and bulk modulus is $\kappa=120.291 \mathrm{~N} / \mathrm{mm}^{2}$. Various values of traction force are chosen for the test (i.e., $F=8,12,16,24 \mathrm{~N} / \mathrm{mm}^{2}$ ). There are five nodal distributions $(10 \times 10,15 \times 15,20 \times 20,25 \times 25$ and $30 \times 30)$ selected to investigate the accuracy of the proposed method in the nonlinear analysis. Fig. 3(b) shows the distribution of $10 \times 10$ scattered nodes and a system of CTM integration points is also displayed (5196 points).
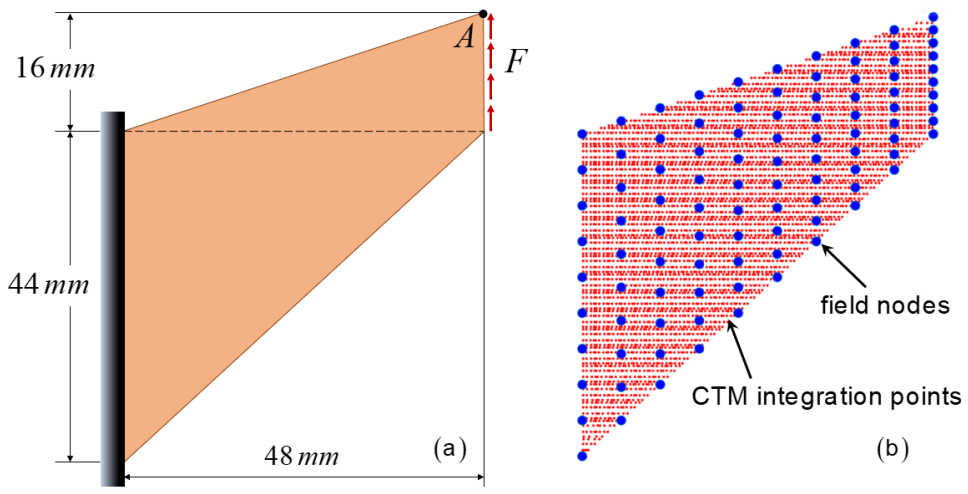

Fig. 3. Cook's membrane problem: geometry and scattered model

The obtained results are verified with the solutions given by variational differential quadrature (VDQ) method [19] that used $23 \times 23$ nodes for the simulation. The present CTM-based RPIM offers acceptable results as the computed vertical displacements at point A (see Fig. 3) are in good agreement with reference solutions [19], which are clearly shown in Fig. 4. The larger the traction force is, the higher value of vertical displacement is obtained. Fig. 5 displays the first Piola Kirchhoff stresses $\left(\mathbf{P}=J \sigma \mathbf{F}^{-T}\right)$ distributions in 
Cook's membrane problem in cases $F=16 \mathrm{~N} / \mathrm{mm}^{2}$ and $F=24 \mathrm{~N} / \mathrm{mm}^{2}, 20 \times 20$ nodes are used for the simulation.

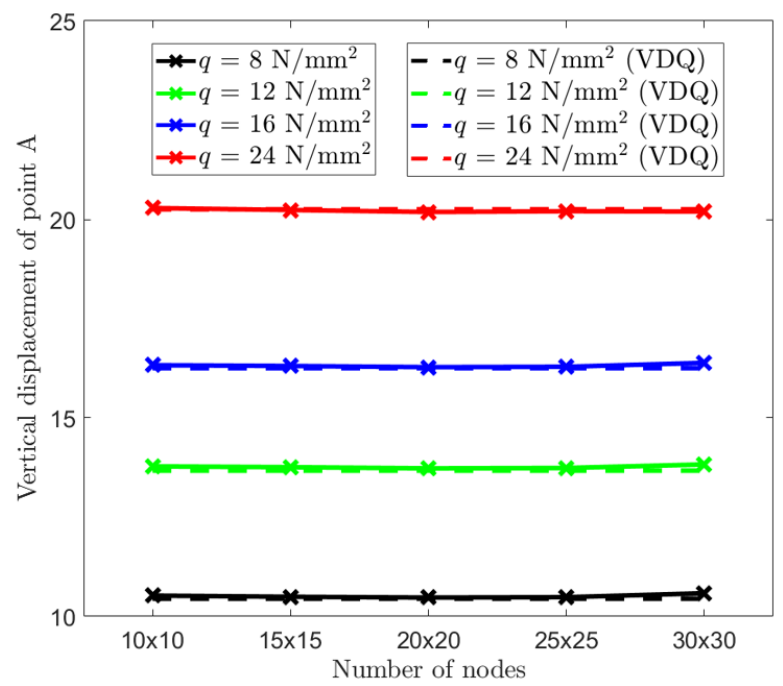

Fig. 4. Vertical displacement of point A with five nodal distributions given by CTM-based RPIM, comparing with VDQ $(23 \times 23$ nodes $)$ [19]
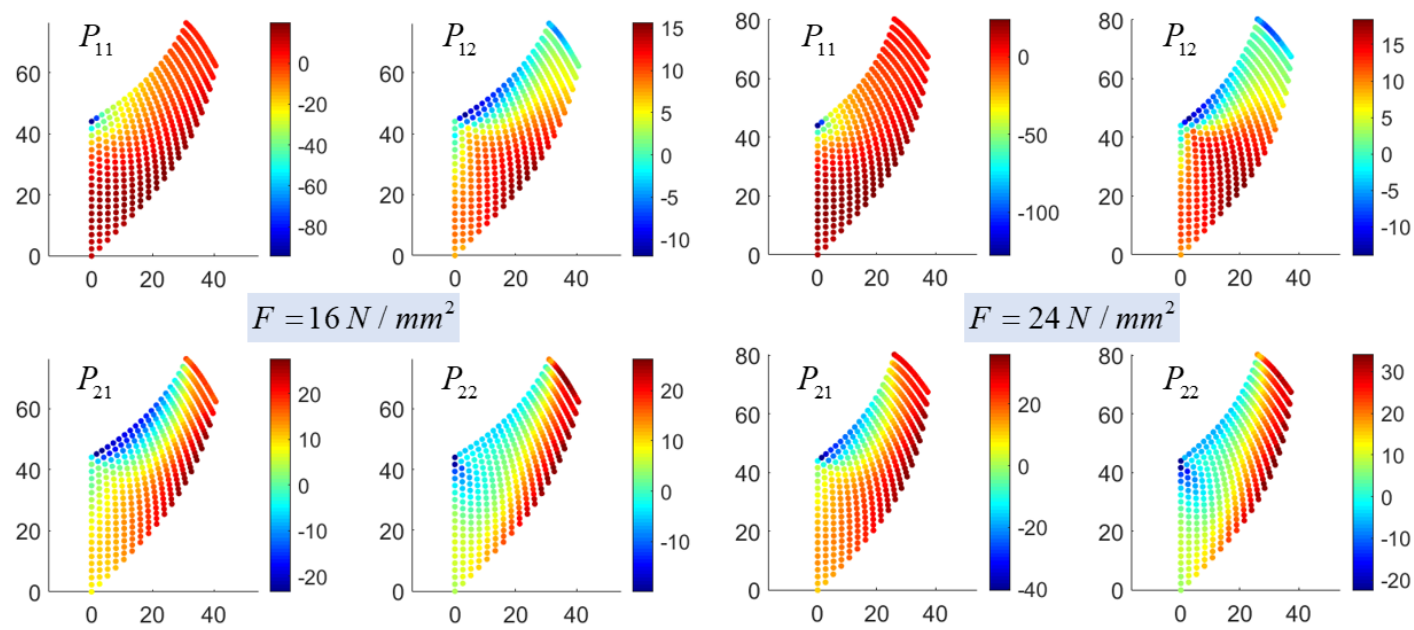

Fig. 5. First Piola Kirchhoff stress distributions in Cook's membrane problem

The computational time estimated for this example by both methods, the conventional Gaussian and CTM techniques, whose computed results are given in Fig. 6, showing the advantage of our development. In this comparison, several nodal densities are used for the Cook's membrane problem with shear force $F=8 \mathrm{~N} / \mathrm{mm}^{2}$ that is divided in 
to 5 load steps. Because Gaussian-based RPIM method uses a system of background cells for integration, so the number of integration points increases significantly when a large number of nodes are used for the interpolation and this essentially requires more time in the computation. As a consequence, the implementation for large deformation problems is effective with the developed CTM-based RPIM. In other words, the computational cost for the present model can be reduced by using the CTM technique.

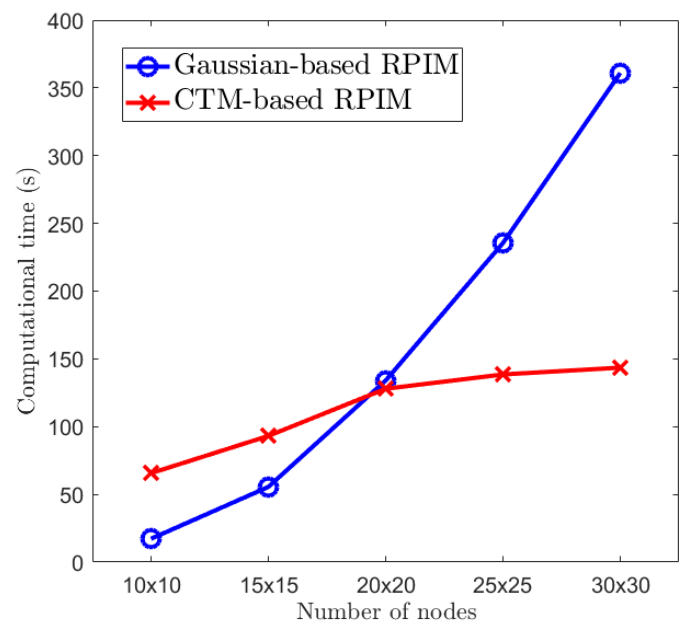

Fig. 6. Computational time comparison between Gaussian-based and CTM-based RPIM methods

Tab. 1 shows the comparison of the vertical displacement of point A between Gaussian-based and CTM-based RPIM methods. A model of $25 \times 25$ nodes are used for both methods and several cases of number of integration points are investigated for CTM approach. The data shows the stable of CTM method and the obtained results from CTM fit well with those from conventional RPIM method although CTM-based RPIM uses less integration points than Gaussian-based method.

Table 1. Vertical displacement of point A given by Gaussian-based and CTM-based RPIM $(25 \times 25$ nodes are used for both methods)

\begin{tabular}{ccccc}
\hline & \multicolumn{4}{c}{ Vertical displacement of point A (mm) } \\
\cline { 2 - 5 }$q\left(\mathrm{~N} / \mathrm{mm}^{2}\right)$ & Gaussian-based & CTM-based \\
\cline { 2 - 5 } & 9216 points & 5196 points & 7112 points & 8604 points \\
\hline 8 & 10.52 & 10.49 & 10.47 & 10.46 \\
12 & 13.80 & 13.74 & 13.70 & 13.72 \\
16 & 16.37 & 16.29 & 16.26 & 16.28 \\
24 & 20.29 & 20.21 & 20.25 & 20.26 \\
\hline
\end{tabular}




\subsection{Inhomogenous compression problem}

The second numerical example is devoted to numerical simulation for a rectangular plate under compression using our CTM-based RPIM. This is to further show the applicability and effectiveness of the developed approach in modeling large deformation problem. The material of plate is compressible hyperelastic, the neo-Hookean model is used and the material parameters are the same with the previous problem. It is assumed that the horizontal displacement of the top edge and vertical displacement of the bottom edge are set zero. A constant distributed load applies on a part of the top edge. The geometry, dimensions and boundary conditions are presented in Fig. 7(a). Because of the symmetry, only a half of the plate is considered in the scattered model, as shown in Fig. 7(b).

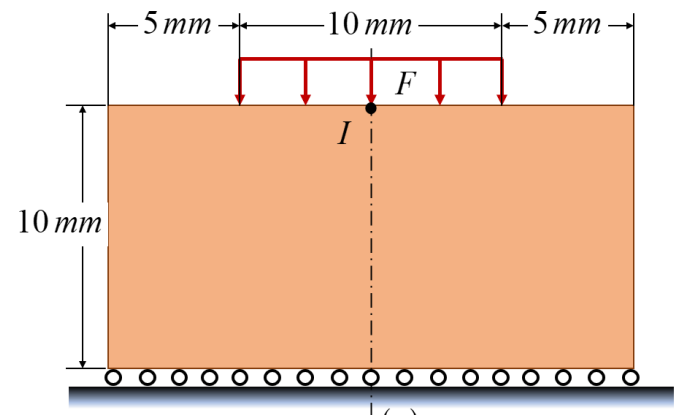

(a)

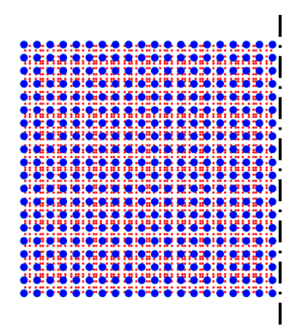

(b)

Fig. 7. Inhomogenous compression problem: geometry and scattered model

Similar to the previous example, to investigate the accuracy and nodal independence of the proposed method, five nodal distributions are selected. The percent of compression is computed at point $I$ with four cases of distributed force $F=50,100,150$ and $200 \mathrm{~N} / \mathrm{mm}^{2}$. Graphs in Fig. 8 (a) show the compression percentages at point $I$ that is obtained by the proposed method and compared with those of VDQ method [19]. The higher values of compression force give larger percents of compression. It is clearly to see that the CTM-based RPIM can give accurate results with less degrees of freedom than the reference method. Fig. 9 depicts the non-linear relationship between the vertical displacement of point $I$ and pressure force $F$, three cases of load steps are also investigated to verify the accuracy of the proposed approach.

The charts in Fig. 10 display the comparison of computational time between the conventional Gaussian and CTM techniques. The distributed force $F=200 \mathrm{~N} / \mathrm{mm}^{2}$ with five load steps is chosen and five cases of nodal distribution are used for the comparison. Once again, the CTM-based RPIM shows its effectiveness in implementation for nonlinear problem. Finally, Fig. 11 represents the deformations in which the color indicates the distribution of norm of the first Piola Kirchhoff stress $\left(\|\mathbf{P}\|=\sqrt{P_{i j} P_{i j}}\right)$ on the models with different values of pressure force. 


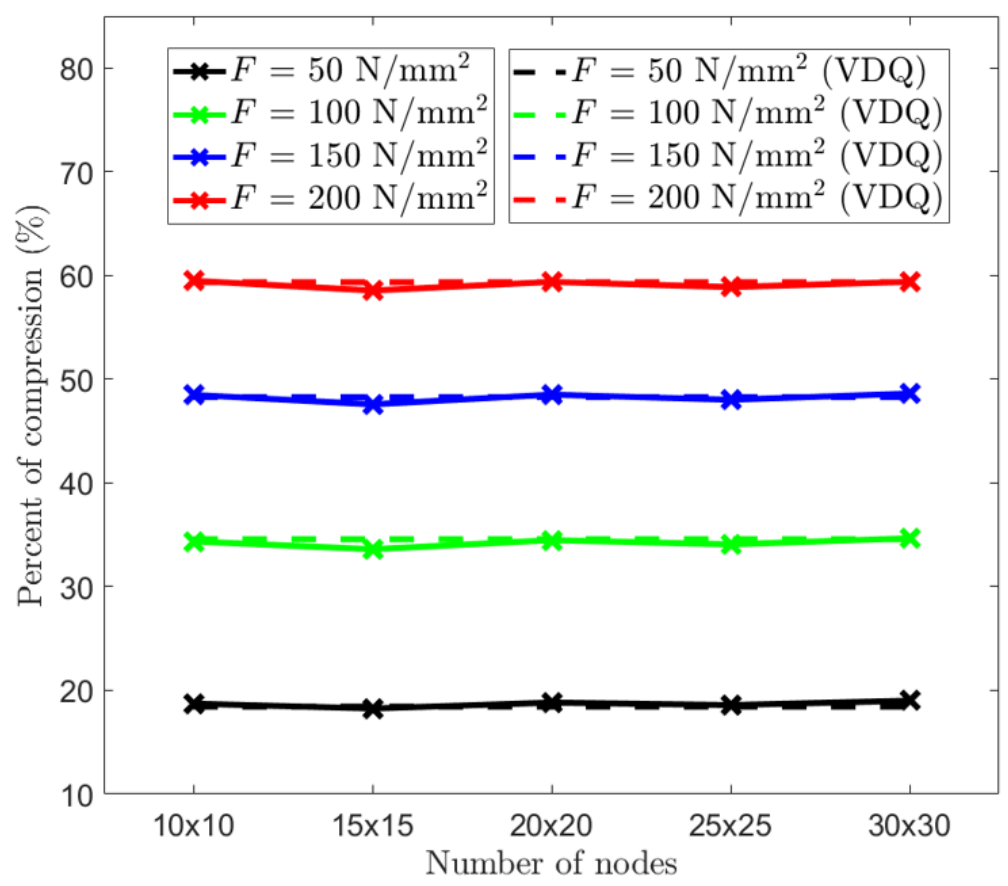

Fig. 8. Percent of compression at point $I$ with five nodal distributions given by CTM-based RPIM, comparing with VDQ $(45 \times 45$ nodes $)$ [19]

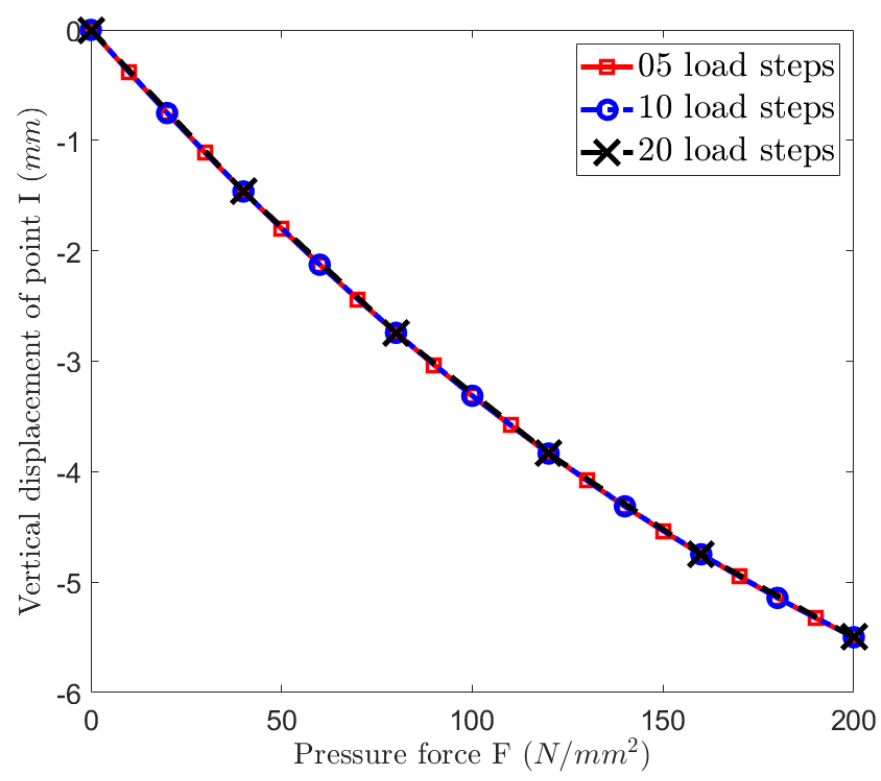

Fig. 9. Force-Displacement diagrams for vertical displacement of point $I$ with different numbers of load steps 


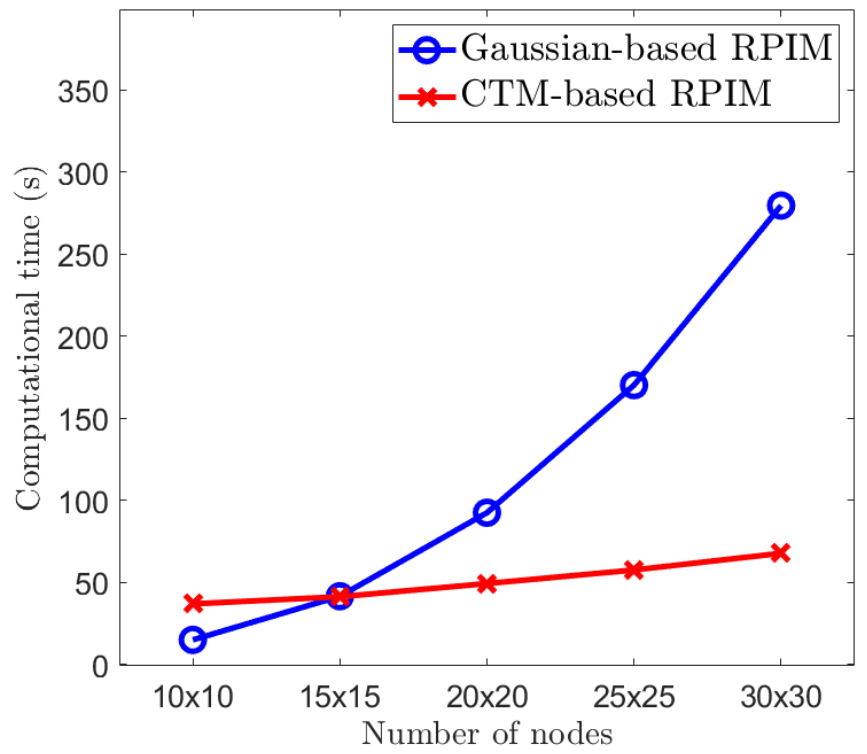

Fig. 10. Computational time comparison between Gaussian-based and CTM-based RPIM methods for inhomogenous compression problem
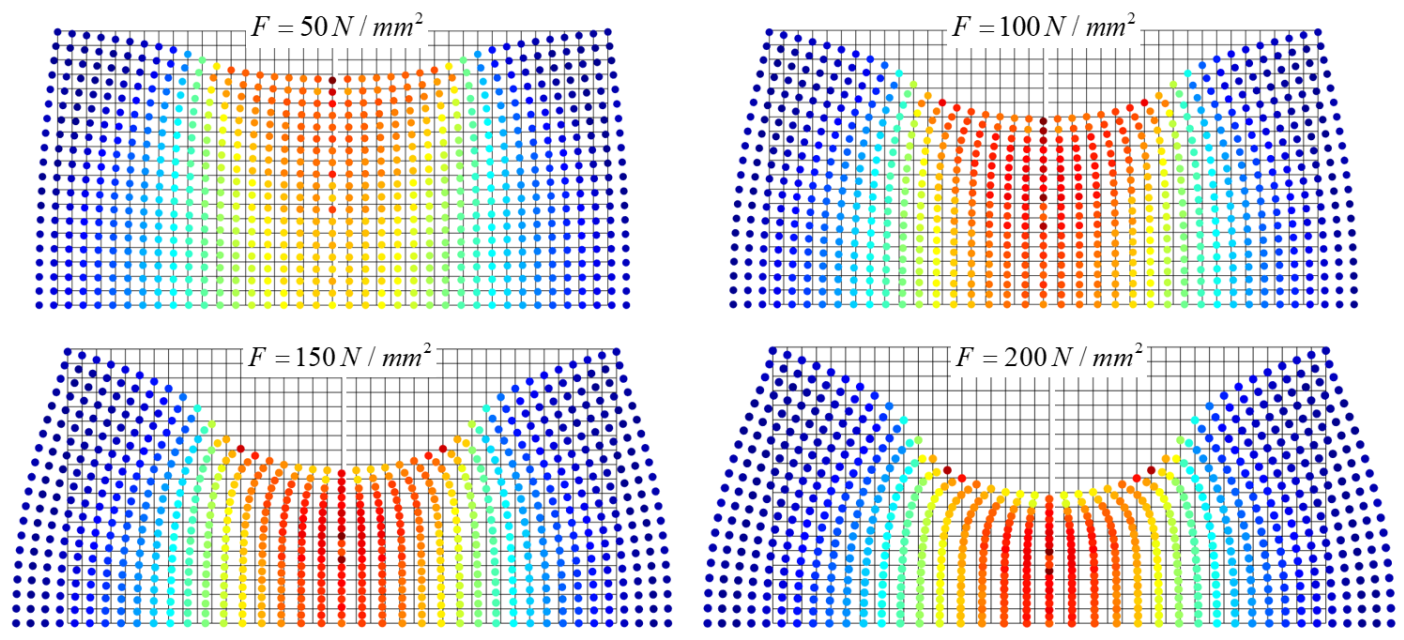

Fig. 11. Norm of first Piola Kirchhoff stress distributions for inhomogenous compression problem (grey grid indicates the initial undeformed configuration of the model)

\subsection{Curved beam under bending}

In the last example, a model of curved beam under bending load is considered. The geometry and boundary conditions are given in Fig. 12. The dimensions are $R_{\text {in }}=9 \mathrm{~mm}$ $R_{\text {out }}=10 \mathrm{~mm}$. The beam is fixed at the lowest edge while its right edge is subject to a distributed force $q$ with angle $\alpha=45^{\circ}$. The material is compressible hyperelastic, the 
properties are the same with the previous examples. Various values of distributed force $q=0.2,0.3,0.4$ and $0.5 \mathrm{~N} / \mathrm{mm}^{2}$ are considered in the simulations. A distribution of $9 \times 101$ nodes and twenty load steps are used for the problem.

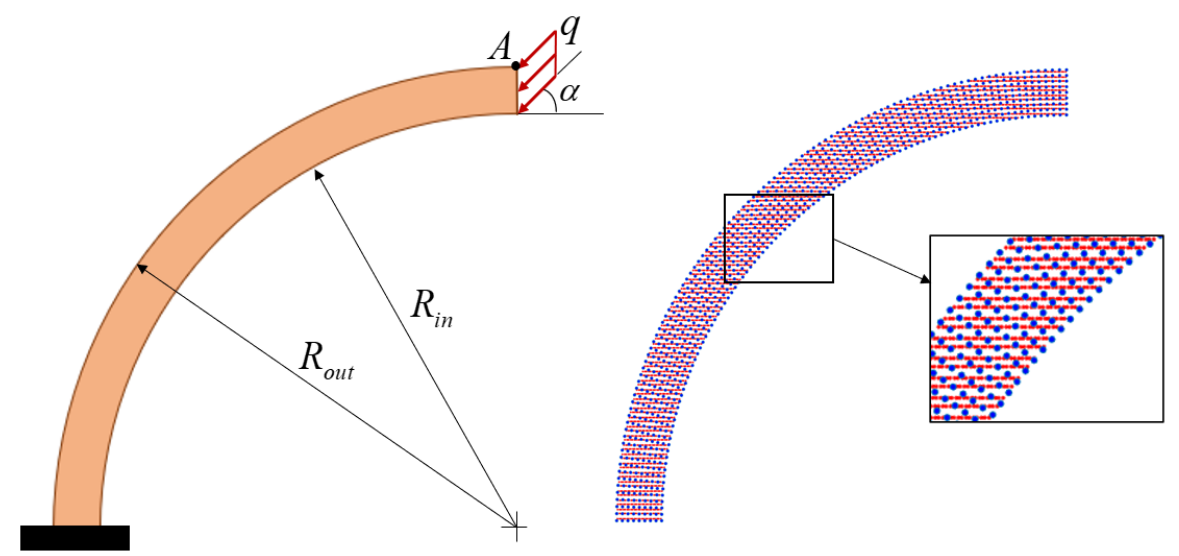

Fig. 12. Curved beam under bending problem: geometry and scattered model

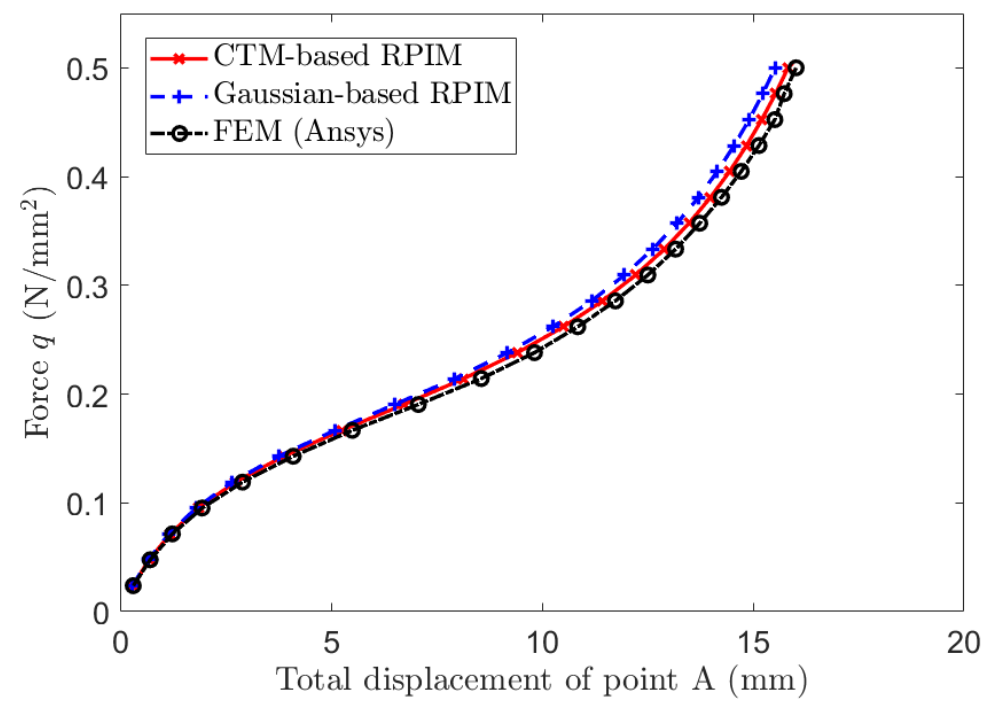

Fig. 13. Force - total displacement diagrams given by CTM-based RPIM, Gaussian-based RPIM and FEM

The charts in Fig. 13 show the non-linear relationship between the external force and total displacement at point A. The obtained results from both CTM-based and Gaussianbased RPIM methods match well with FEM solutions given by ANSYS program. Finally, the distributions of total displacement in the curved beam in four cases of distributed force $q$ are displayed in Fig. 14. 

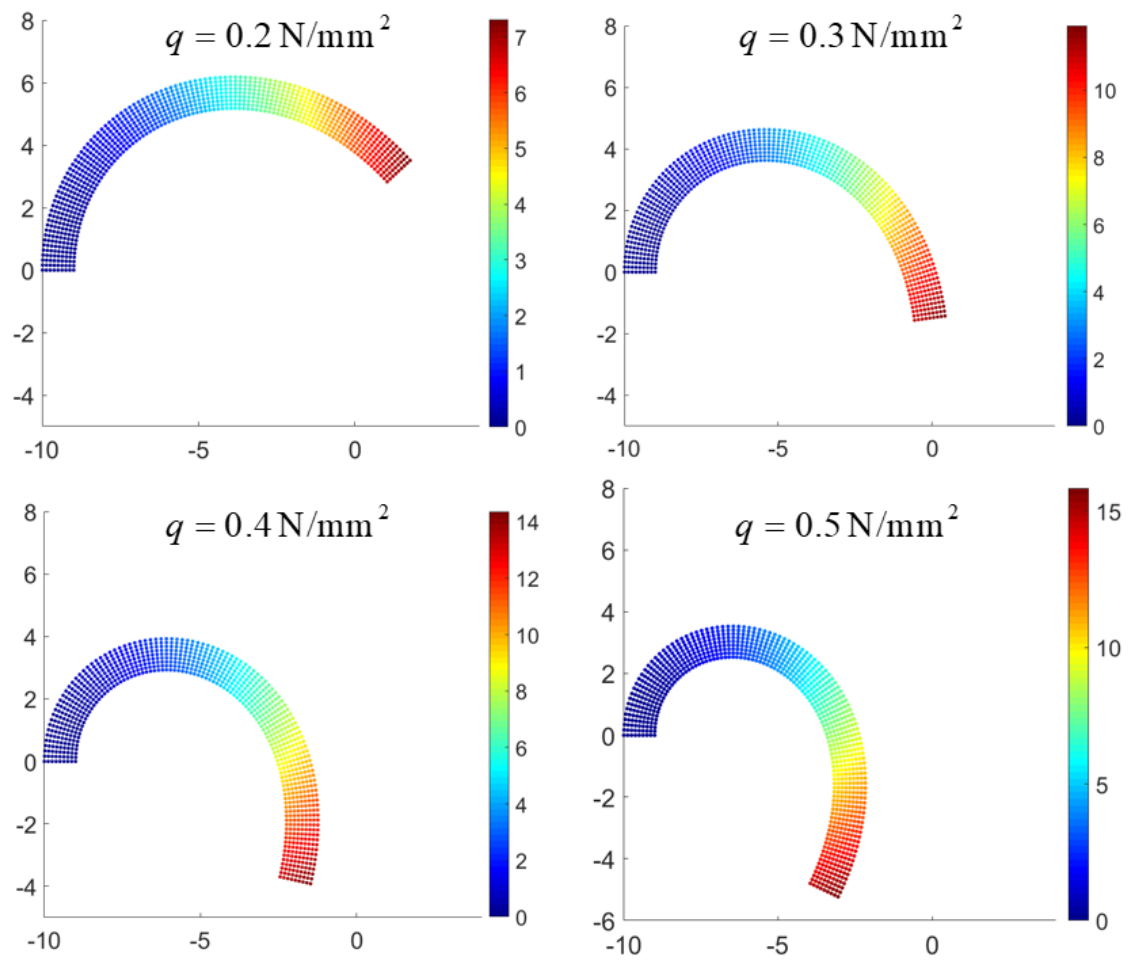

Fig. 14. Distributions of total displacement in the curved beam

\section{CONCLUSIONS}

In this paper, we have developed an enhanced and effective CTM-based RPIM for finite deformation analysis of compressible hyperelastic materials. The CTM integration technique is used as alternative to traditional Gaussian quadrature method and this makes the meshless RPIM method become independent from the 2D integration cells. There is no $2 \mathrm{D}$ meshing algorithm is required to generate the background cells. Three numerical examples are performed and the obtained results are verified with reference solution. The proposed method has shown good performance in both accuracy and computation cost for large deformation problem. It is promising for further investigation on behavior of hyperelastic materials such as dynamic problems and fracture analysis. However, in this study, only regular nodal distributions are considered so the variations of nodal density would be taken in account in the future works. Moreover, because CTM method is being developed, there is still not any general algorithm to generate CTM integration points for every type of geometry. These drawbacks should be improved in next studies.

\section{ACKNOWLEDGMENT}

This research is funded by Vietnam National Foundation for Science and Technology Development (NAFOSTED) under grant number 107.02-2019.327. 
Nha Thanh Nguyen, Minh Ngoc Nguyen, Thien Tich Truong, Tinh Quoc Bui

\section{REFERENCES}

[1] A. M. Maniatty, Y. Liu, O. Klaas, and M. S. Shephard. Higher order stabilized finite element method for hyperelastic finite deformation. Computer Methods in Applied Mechanics and Engineering, 191, (13-14), (2002), pp. 1491-1503. https://doi.org/10.1016/s0045-7825(01)00335-8.

[2] A. A. Ramabathiran and S. Gopalakrishnan. Automatic finite element formulation and assembly of hyperelastic higher order structural models. Applied Mathematical Modelling, 38, (11-12), (2014), pp. 2867-2883. https://doi.org/10.1016/j.apm.2013.11.021.

[3] A. Nomoto, H. Yasutaka, S. Oketani, and A. Matsuda. 2-dimensional homogenization FEM analysis of hyperelastic foamed rubber. Procedia Engineering, 147, (2016), pp. 431-436. https://doi.org/10.1016/j.proeng.2016.06.335.

[4] A. Angoshtari, M. F. Shojaei, and A. Yavari. Compatible-strain mixed finite element methods for 2D compressible nonlinear elasticity. Computer Methods in Applied Mechanics and Engineering, 313, (2017), pp. 596-631. https://doi.org/10.1016/j.cma.2016.09.047.

[5] T. Belytschko, Y. Y. Lu, and L. Gu. Element-free Galerkin methods. International Journal for Numerical Methods in Engineering, 37, (2), (1994), pp. 229-256. https://doi.org/10.1002/nme.1620370205.

[6] T. Belytschko, Y. Krongauz, D. Organ, M. Fleming, and P. Krysl. Meshless methods: An overview and recent developments. Computer Methods in Applied Mechanics and Engineering, 139, (1), (1996), pp. 3-47. https://doi.org/10.1016/s0045-7825(96)01078-x.

[7] N. S. Atluri and T. Zhu. A new meshless local Petrov-Galerkin (MLPG) approach in computational mechanics. Computational Mechanics, 22, (2), (1998), pp. 117-127. https://doi.org/10.1007/s004660050346.

[8] J. G. Wang and G. R. Liu. A point interpolation meshless method based on radial basis functions. International Journal for Numerical Methods in Engineering, 54, (11), (2002), pp. 1623-1648. https://doi.org/10.1002/nme.489.

[9] L. Gu. Moving kriging interpolation and element-free Galerkin method. International Journal for Numerical Methods in Engineering, 56, (1), (2002), pp. 1-11. https://doi.org/10.1002/nme.553.

[10] Y. T. Gu, Q. X. Wang, and K. Y. Lam. A meshless local Kriging method for large deformation analyses. Computer Methods in Applied Mechanics and Engineering, 196, (9-12), (2007), pp. 16731684. https://doi.org/10.1016/j.cma.2006.09.017.

[11] D. A. Hu, S. Y. Long, X. Han, and G. Y. Li. A meshless local Petrov-Galerkin method for large deformation contact analysis of elastomers. Engineering Analysis with Boundary Elements, 31, (7), (2007), pp. 657-666. https://doi.org/10.1016/j.enganabound.2006.11.005.

[12] D. Hu, Z. Sun, C. Liang, and X. Han. A mesh-free algorithm for dynamic impact analysis of hyperelasticity. Acta Mechanica Solida Sinica, 26, (4), (2013), pp. 362-372. https://doi.org/10.1016/s0894-9166(13)60033-6.

[13] Y. Zhang, W. Ge, Y. Zhang, Z. Zhao, and J. Zhang. Topology optimization of hyperelastic structure based on a directly coupled finite element and elementfree Galerkin method. Advances in Engineering Software, 123, (2018), pp. 25-37. https://doi.org/10.1016/j.advengsoft.2018.05.006.

[14] E. Khosrowpour, M. R. Hematiyan, and M. Hajhashemkhani. A strong-form meshfree method for stress analysis of hyperelastic materials. Engineering Analysis with Boundary Elements, 109, (2019), pp. 32-42. https://doi.org/10.1016/j.enganabound.2019.09.013.

[15] A. Khosravifard and M. R. Hematiyan. A new method for meshless integration in 2D and 3D Galerkin meshfree methods. Engineering Analysis with Boundary Elements, 34, (1), (2010), pp. 30-40. https://doi.org/10.1016/j.enganabound.2009.07.008. 
[16] A. Khosravifard, M. R. Hematiyan, and L. Marin. Nonlinear transient heat conduction analysis of functionally graded materials in the presence of heat sources using an improved meshless radial point interpolation method. Applied Mathematical Modelling, 35, (9), (2011), pp. 4157-4174. https://doi.org/10.1016/j.apm.2011.02.039.

[17] T. Q. Bui, A. Khosravifard, C. Zhang, M. R. Hematiyan, and M. V. Golub. Dynamic analysis of sandwich beams with functionally graded core using a truly meshfree radial point interpolation method. Engineering Structures, 47, (2013), pp. 90-104. https://doi.org/10.1016/j.engstruct.2012.03.041.

[18] N. T. Nguyen, T. Q. Bui, M. N. Nguyen, and T. T. Truong. Meshfree thermomechanical crack growth simulations with new numerical integration scheme. Engineering Fracture Mechanics, (2020), p. 107121. https://doi.org/10.1016/j.engfracmech.2020.107121.

[19] R. Hassani, R. Ansari, and H. Rouhi. Large deformation analysis of 2D hyperelastic bodies based on the compressible nonlinear elasticity: A numerical variational method. International Journal of Non-Linear Mechanics, 116, (2019), pp. 39-54. https://doi.org/10.1016/j.ijnonlinmec.2019.05.003.

[20] J. P. Pascon. Large deformation analysis of plane-stress hyperelastic problems via triangular membrane finite elements. International Journal of Advanced Structural Engineering, 11, (3), (2019), pp. 331-350. https://doi.org/10.1007/s40091-019-00234-w.

[21] G. R. Liu, G. Y. Zhang, Y. T. Gu, and Y. Y. Wang. A meshfree radial point interpolation method (RPIM) for three-dimensional solids. Computational Mechanics, 36, (6), (2005), pp. 421-430. https://doi.org/10.1007/s00466-005-0657-6.

[22] G. R. Liu. Mesh free methods - Moving beyon the finite element method. CRC Press, (2009).

[23] P. Wriggers. Nonlinear Finite Element Methods. Springer Berlin Heidelberg, (2008). https://doi.org/10.1007/978-3-540-71001-1.

[24] H. D. Huynh, P. Tran, X. Zhuang, and H. Nguyen-Xuan. An extended polygonal finite element method for large deformation fracture analysis. Engineering Fracture Mechanics, 209, (2019), pp. 344-368. https://doi.org/10.1016/j.engfracmech.2019.01.024. 